This document is the accepted manuscript version of the following article:

Boese1, L. F. (2015). Effect of plasticizers on the barrier and

mechanical properties of biomimetic composites of chitosan and

clay. Carbohydrate Polymers, 115, 356-363. https://

doi.org/10.1016/j.carbpo1.2014.08.064

This manuscript version is made available under the CC-BY-NC-ND

4.01 icense http://creativecommons.org/1icenses/by-nc-nd/4.

\title{
Effect of plasticizers on the barrier and mechanical properties of biomimetic composites of chitosan and clay
}

\author{
Luciano F. Boesel
}

Empa - Swiss Federal Laboratories for Materials Science and Technology, Laboratory for Bioactive Materials and Laboratory for Protection and Physiology, Lerchenfeldstrasse 5, 9014, St. Gallen, Switzerland.

E-mail: Luciano.Boesel@empa.ch

\begin{abstract}
Biomimetic composites of polymer and clay are known to possess high mechanical strength and excellent barrier properties. However, the ductility of these composites is always low. One way to overcome this limitation consists on the incorporation of plasticizers which work effectively for the pure polymer. It is shown here that an imidazolium-based ionic liquid is a more effective plasticizer for chitosan, in terms of suppression of the glass transition temperature and mechanical properties, than the more commonly used glycerol. This might result from the ionic interaction between both species that is not present when glycerol is used as the plasticizer. Biomimetic composites of chitosan and clay were also prepared containing these two plasticizers. A similar effect on the mechanical properties was observed as for the pure polymer, that is, even at a very low $(\sim 12 \%)$ final concentration, the ionic liquid was a better plasticizer than glycerol, being able to double the ductility of the composites. Regarding barrier properties, no difference was observed between the original and the plasticized composites. This study thus shows that the incorporation of plasticizers is an effective method to improve the flexibility of biomimetic composites without deleterious effect on their excellent barrier properties.
\end{abstract}

Keywords: layered structures, nanoclays, transport properties, biopolymers, nanocomposites, ionic liquids

\section{Introduction}

The use of biological materials as source of inspiration to design innovative materials has attracted increased interest in recent years and this class 
of materials, known as biomimetic or bioinspired materials are now established as an important field of research in materials science. (Zollfrank et al., 2012) Among the diverse structures found in nature, the one of nacre, a natural composite found in mollusc shells, has served as model to the material science community, because it possesses an optimal combination of soft and hard materials. (Bonderer et al., 2008; Espinosa et al., 2009; Walther et al., 2010) It consists of a layered arrangement of inorganic platelets bonded by a small amount (about $5 \%$ to $10 \%$ ) of proteins and polysaccharides. This structure imparts a very high mechanical property to the natural composite, (Bonderer et al., 2008) which has been mimicked by a variety of methods such as layer-by-layer or repeated spin coating processes. (Bonderer et al., 2008; Priolo et al., 2010a; Walther et al., 2010) Due to the high inorganic content and layered arrangement of the inorganic platelets, this structure also performs well as a barrier layer against the permeation of gases or vapours. Accordingly, a great deal of effort has been dedicated to improve the barrier properties of both synthetic and natural polymers (e. g. Priolo et al. (2010a,b)). Although the optimization of the interface between the polymer and the particle and of the inorganic content has led to dramatic improvements of mechanical and barrier properties of several materials, the requirement for dozens of alternate dip- or spin- coating processes, washing and drying steps, makes this methodology less interesting for large-scale industrial production.

Recently, an alternative technique has been developed that allows high throughput fabrication of such biomimetic composites in one-step process. (Walther et al., 2010) In this method, a polymer solution and a clay dispersion are mixed allowing the polymer to adsorb on the clay surface. The mixture is then applied in a single step through doctor-blading to allow the preparation of self-supporting films or coatings onto another polymer substrate. Although the ordering and alignment of clay platelets in this case is worse than that obtained in a layer-by-layer process, (Walther et al., 2010) the simplicity of the application makes this process well suitable for industrial purposes.

This methodology has been expanded to biobased and biodegradable polymers such as chitosan (Yao et al., 2010), PLA (Boesel et al., 2013), and cellulose (Liu et al., 2011). A common problem with these composites, however, is their brittleness. Due to the high amount of clay platelets (50-80\%), the elongation at break usually falls below $2 \%$, even if the specimens are kept at $50 \%$ relative humidity. In the dry state, the ductility of the composites is much lower. An usual way of increasing the ductility of polymers or composites is through the addition of plasticizers to increase the free vol- 
ume and/or decrease the intermolecular interactions of polymer chains. If such plasticizer effect could be maintained in the biomimetic composites, a more ductile, though still high barrier material could be developed as barrier coatings for biopolymers.

In this article, the one-step process was applied for the preparation of highly loaded chitosan/clay composites. This method had already been applied before (Yao et al., 2010) to chitosan, but the materials were too brittle and no barrier properties were reported. I thus prepared similar composites containing plasticizers, in order to evaluate the influence of such plasticizers on the mechanical and barrier properties of chitosan bionanocomposites.

\section{Results and Discussion}

\subsection{Characterization of plasticized films of chitosan}

While glycerol is a well known plasticizer for polysaccharide materials such as starch and chitosan (Epure et al., 2011), ionic liquids have only recently been reported as an efficient plasticizer for starch (Ning et al., 2009; Sankri et al., 2010). To the best of my knowledge, no study has been reported on the plasticization effects of IL on chitosan, although one may assume they would be similar as those observed for starch. In this study, both kinds of plasticizers were used to compare their effects on the properties of chitosan and on chitosan/clay composites.

Towards this objective, I initially prepared mixtures of chitosan containing $30 \%$ glycerol (Gly30), or $30 \%$ or $10 \%$ of IL (IL30 and IL10, respectively). Next, I performed an extended characterization of chitosan plasticized with IL or glycerol using SEM, FTIR, DMTA, and barrier and mechanical properties.

Figure 1 shows SEM micrographs of cryogenically fractured surfaces of the 4 materials. Three different behaviours can be distinguished: a flat fracture surface, characteristic of brittle materials, on pure chitosan; an inhomogeneous, with intermediate roughness surface on chitosan plasticized with $30 \%$ glycerol or $10 \% \mathrm{IL}$; and a rough surface in the case of chitosan plasticized with $30 \%$ IL, characteristic of very ductile materials. Noteworthy, all surfaces were created under cryogenic fracture; therefore, even ductile materials could appear brittle. The fact that IL30 presented such a rough surface under these conditions clearly suggests a very high plasticizing effect of IL.

These findings were corroborated by mechanical tests, as shown in Table 1. To eliminate the effect of water as a plasticizer, tests were performed 


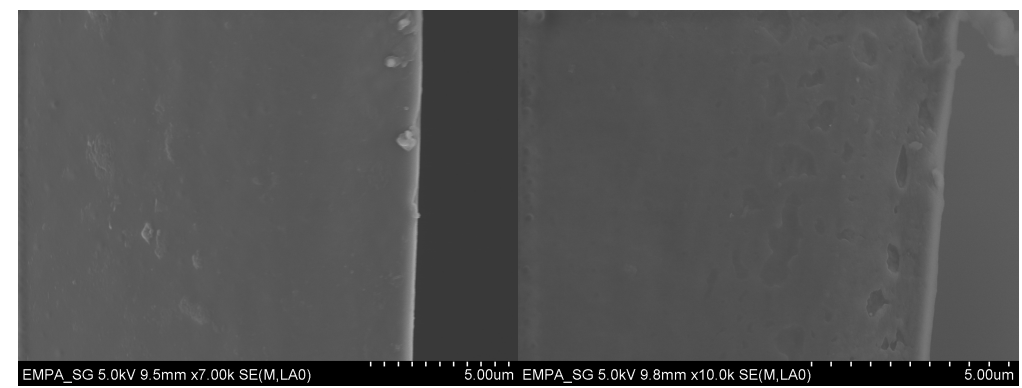

(a)

(b)

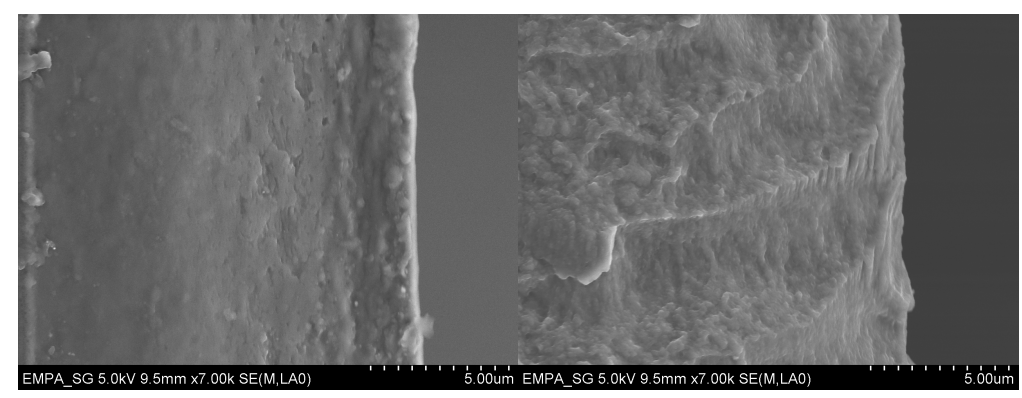

(c)

(d)

Figure 1: SEM images of the cross section of a pure chitosan film (a), and chitosan plasticized with: (b) $30 \%$ glycerol, (c) $10 \%$ IL, and (d) $30 \%$ IL. 
on vacuum dried specimens $\left(60{ }^{\circ} \mathrm{C} 24 \mathrm{~h}\right)$ in addition to the standard conditions (equilibration at $50 \% \mathrm{RH}$ ). In any case, chitosan is a strong and stiff material, with a medium degree of ductility. Specifically, the vacuum dried material presented very high tensile strength. Both glycerol and IL are effective plasticizers for chitosan, but the latter is much more efficient (e.g., in terms of $E_{t}$ or $\epsilon_{b}$ ). The pattern seen in the SEM micrographs is corroborated by the mechanical properties measured from the dried samples, that is, the addition of $30 \%$ glycerol or $10 \%$ IL resulted in materials with very similar mechanical properties (no significant differences were found for the four properties displayed in Table 1). Moreover, when comparing the properties of Gly30 tested in dry conditions with those of chitosan in wet conditions (that is, water plasticized chitosan), one clearly notices that the properties lay in the same order of magnitude, even though the amount of glycerol $(30 \%)$ is much higher than that of water (ca. $17 \%)$. In fact, the addition of glycerol also increased the water uptake as shown in Table 2 and the water sensitivity as shown in Figure S1: noticeable decreases on the mechanical properties were observed with small variations in water content (19-23\%). None of these effects were observed with IL, confirming that the high efficacy of IL is due to an inherently better plasticization mechanism and not to an increase in the water uptake or water sensitivity of chitosan. Figures S2a and S2b display representative curves of the films under dry and wet conditions. The transition in the mode of deformation and fracture is clearly evident in these figures when going from chitosan to IL30.

Plasticizers usually act to decrease the intermolecular interaction of polymer chains, either by increasing the free volume of polymers or by interacting directly with functional groups in their backbone. In either case the mobility of the amorphous place (where plasticizers preferentially act) will be increased, which translates into a decrease of the glass transition temperature $\left(T_{g}\right)$. I used DMTA in tensile mode to determine the $T_{g}$ of the four compositions. Figures 2 and S3 show a representative curve for each material. The behaviour of IL30 differs considerably from the other three materials: not only is the peak corresponding to $T_{g}$ at a much lower temperature, but also its maximum is much higher, a sign of an increased liquid-like behaviour of the polymer chains in the amorphous phase. The addition of glycerol or $10 \% \mathrm{IL}$, on the other hand, provoked only a minor decrease in the $T_{g}$ and a minor increase of the peak intensity; moreover, the extent of $T_{g}$ depression was approximately the same for both plasticizers $\left(\sim 5^{\circ} \mathrm{C}\right)$, thus confirming the low efficiency of glycerol as plasticizer. The curve of Gly30 shows a second broad peak at $\sim 32{ }^{\circ} \mathrm{C}$ which was assigned to the $T_{g}$ of glycerol. This transition has been determined for pure glycerol at 
Table 1: Mechanical properties of plasticized chitosan films

\begin{tabular}{c|c|c|c|c}
\hline Sample & $E_{t}(\mathrm{MPa})$ & $\sigma_{y}(\mathrm{MPa})$ & $\sigma_{t}(\mathrm{MPa})$ & $\epsilon_{b}(\%)$ \\
\hline \multicolumn{5}{c}{ At $50 \% \mathrm{RH}$} \\
\hline Chitosan & $2,6 \pm 0,2^{a}$ & $54 \pm 3^{a}$ & $68 \pm 13^{a}$ & $24 \pm 9^{a}$ \\
Gly30 & $0,81 \pm 0,22^{b}$ & $20 \pm 5^{b}$ & $44 \pm 11^{b}$ & $58 \pm 11^{b}$ \\
IL30 & $0,084 \pm 0,020^{c}$ & $3,1 \pm 1,0^{c}$ & $34 \pm 6^{b}$ & $(1,3 \pm 0,1) \times 10^{2, c}$ \\
\hline \multicolumn{5}{c}{ Vacuum dried } \\
\hline Chitosan & $3,6 \pm 0,7^{a}$ & $(1,0 \pm 0,2) \times 10^{2, a}$ & $(1,2 \pm 0,2) \times 10^{2, a}$ & $18 \pm 8^{a}$ \\
Gly30 & $1,6 \pm 0,7^{b}$ & $41 \pm 20^{b}$ & $79 \pm 13^{b}$ & $46 \pm 9^{b}$ \\
IL10 & $1,8 \pm 0,5^{b}$ & $51 \pm 17^{b}$ & $84 \pm 17^{b}$ & $43 \pm 1^{b}$ \\
IL30 & $0,12 \pm 0,03^{c}$ & $4,4 \pm 0,8^{c}$ & $40 \pm 5^{c}$ & $(1,1 \pm 0,2) \times 10^{2, c}$ \\
\hline
\end{tabular}

Superscripts "a, b, c" identify samples (in the same column and for each of the two storing conditions) which do not show significant differences for the corresponding property at $p<0,01$.

Table 2: Water uptake (WU), permeability, and position of the amide II band of plasticized and pure chitosan

\begin{tabular}{c|c|c|c|c}
\hline Sample & $\begin{array}{c}\text { WU } \\
(\%)\end{array}$ & $\begin{array}{c}\mathrm{P}_{W V} \\
*\end{array}$ & $\begin{array}{c}\mathrm{P}_{O_{2}} \\
*\end{array}$ & $\begin{array}{c}\text { Amide II band } \\
\left(\mathrm{cm}^{-1}\right)\end{array}$ \\
\hline Chitosan & $16,6 \pm 0,9$ & $12 \pm 4$ & $1,2 \pm 0,5$ & 1534 \\
Gly30 & $20,8 \pm 1,4^{a}$ & $15 \pm 3$ & $6,9 \pm 2,4^{a}$ & 1543 \\
IL10 & - & $14 \pm 2$ & $8,6 \pm 1,4^{b}$ & 1544 \\
IL30 & $17,1 \pm 1,5$ & $18 \pm 5^{a}$ & $(2,0 \pm 0,4) \times 10^{2, b}$ & 1563 \\
\hline
\end{tabular}

* Units: $\mathrm{P}_{W V}$ in $\left[10^{-14} \mathrm{~kg} \cdot \mathrm{m} /\left(\mathrm{m}^{2} \cdot s \cdot \mathrm{Pa}\right)\right]$, and $\mathrm{P}_{\mathrm{O}_{2}}$ in $\left[10^{-20} m^{3} \cdot m /\left(m^{2} \cdot s \cdot P a\right)\right]$.

${ }^{a}$ Value significantly different (at $p<0,05$ ) from that of chitosan.

${ }^{b}$ Value significantly different $($ at $p<0,01)$ from that of chitosan. 


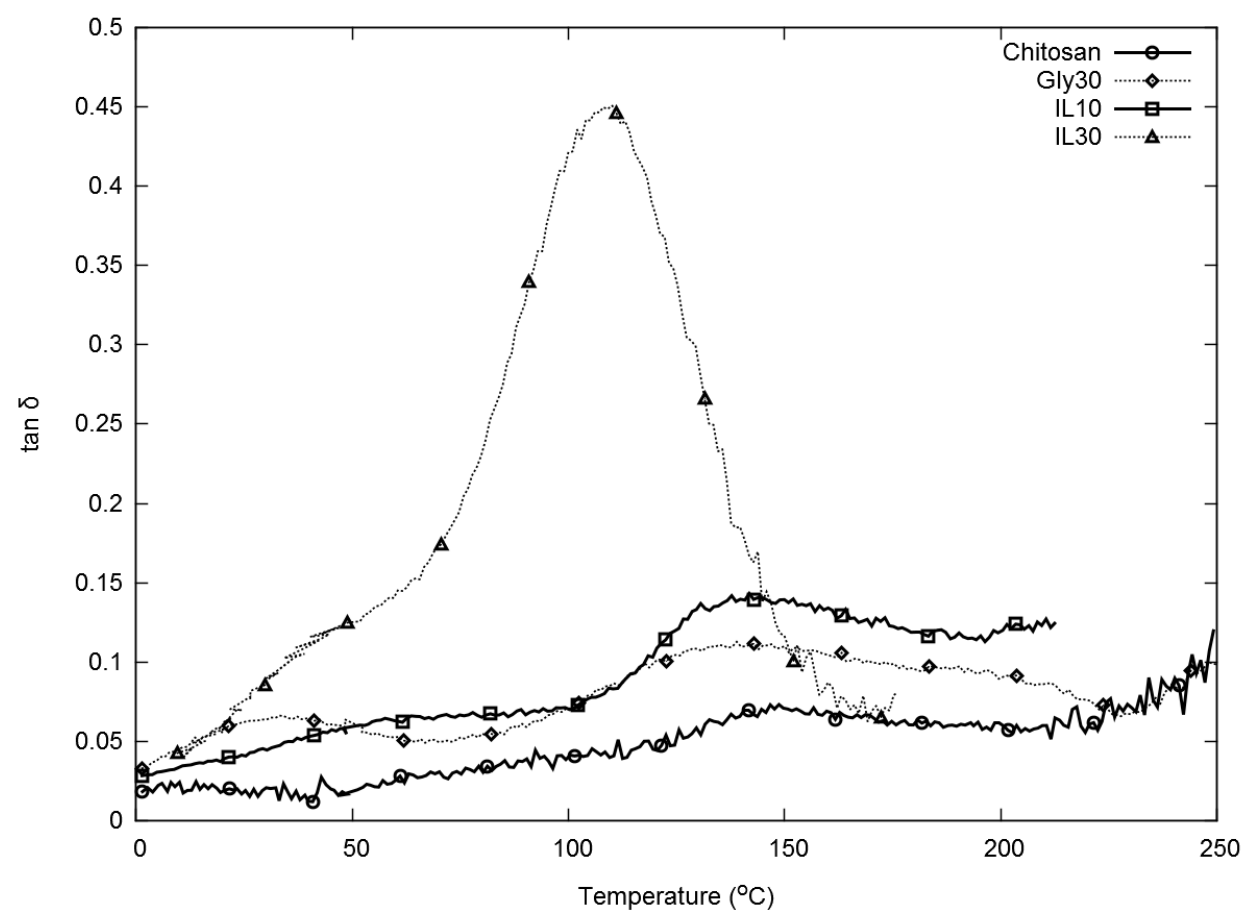

Figure 2: Representative DMTA curves of pure and plasticized films of chitosan. The $T_{g}$ of the films is: Chitosan $\approx 150{ }^{\circ} \mathrm{C}$, Gly30 and IL10 $\approx$ $145{ }^{\circ} \mathrm{C}$, and IL30 $=109{ }^{\circ} \mathrm{C}$. See also Figure S3 for an expanded view of Chitosan, Gly30, and IL30.

$\sim-83{ }^{\circ} \mathrm{C}$. However, in the chitosan/glycerol materials, the $T_{g}$ increases due to a decrease in glycerol mobility, as the chains mobility are hindered by the surrounding chitosan molecules. The same behaviour was shown by Quijada-Garrido et al. (2007).

Another effect of the increased mobility of the chains in the amorphous phase is the increased permeability to gases and vapours. Since permeation does not occur through crystal phases, and as the increase in chain mobility would facilitate diffusion of the permeant, the plasticizing effect should also have an impact on the permeability of the films. Table 2 lists the results of oxygen and water vapour permeability. A pattern similar to the one seen for the mechanical properties or $T_{g}$ is observed here, especially for oxygen permeability. The effect is much less evident for water vapour permeability, 
as chitosan itself is very sensitive to water and, as seen for the mechanical properties, water plasticizes the matrix. Therefore, the effect of additional plasticization would be mostly masked by the effect of water itself. Nonetheless, and although the absolute difference was small, IL30 was significantly more permeable than chitosan, once more confirming the high plasticizing potential of this substance. As both oxygen and water vapour permeation were changed, it may be assumed that the effect is mainly due to a physical factor (that is, increase in diffusion due to increased chain mobility) rather than to a chemical one (increase in solubility due to the presence of the plasticizer). In the latter case one would expect to find mostly an increase in the water vapour permeation, because both plasticizers are hydrophilic; however, in my samples the opposite was true.

FTIR was used to understand how the plasticizers were interacting with chitosan. Figure 3 shows the spectra of pure and plasticized chitosan. Chitosan prepared as described in Section 4 displays the typical bands for this material, with some differences: the wide absorption band from the stretching vibration of $\mathrm{OH}$ or $\mathrm{NH}$ groups is shifted towards $\sim 3180 \mathrm{~cm}^{-1}$, the amide I band (stretching vibrations of carbonyl group $\mathrm{C}=\mathrm{O}$ ) decreased to $\sim 1635 \mathrm{~cm}^{-1}$, and the amide II band (bending absorption of $\mathrm{N}-\mathrm{H}$ ) appeared at $\sim 1540 \mathrm{~cm}^{-1}$. For comparison, the amide I and II bands of neutralized chitosan appeared at $\sim 1650 \mathrm{~cm}^{-1}$ and $\sim 1580 \mathrm{~cm}^{-1}$, respectively. Changes on the other bands of chitosan were not observed, as shown in Figure 3. The shifting towards low wavenumbers of the amide bands and the NH vibrations has been well described in the literature (Matet et al., 2013; Osman and Arof, 2003) and is due to an interaction between the $-\mathrm{COO}^{-}$in acetic acid and $-\mathrm{NH}_{3}^{+}$in chitosan.

The addition of $30 \%$ of IL as plasticizer caused clear changes in the chitosan spectrum (Figure 3): the appearance of several bands between 2800$3150 \mathrm{~cm}^{-1}$ (assigned to $\mathrm{CH}_{3}, \mathrm{CH}_{2}$, and $\mathrm{CH}$ stretching from the BMIM$\mathrm{Cl}$ (Jeon et al., 2008)) as well as a new band at $\sim 1460 \mathrm{~cm}^{-1}$ (assigned to the $\mathrm{CH}_{2}$ deformation vibration of the alkyl chain of BMIM-Cl). More important, however, are the changes in the typical bands of chitosan discussed previously (see Table 2): all of them shifted back to their "original" positions (that is, those of chitosan without acetic acid) with the addition of $30 \%$ IL. None of these new bands or band shifting were observed when IL was added at $10 \%$. Sankri et al. described that each ion pair $\mathrm{Cl}^{-} / \mathrm{BMIM}^{+}$forms complex interactions with a C-O-H group of starch so that hydrogen bonding with both water molecules or other $\mathrm{OH}$ groups of starch are reduced or not possible any longer.(Sankri et al., 2010) This leads to a shift towards higher wavenumbers of the $\mathrm{OH}$ stretching vibrations,(Sankri et al., 2010) similar 


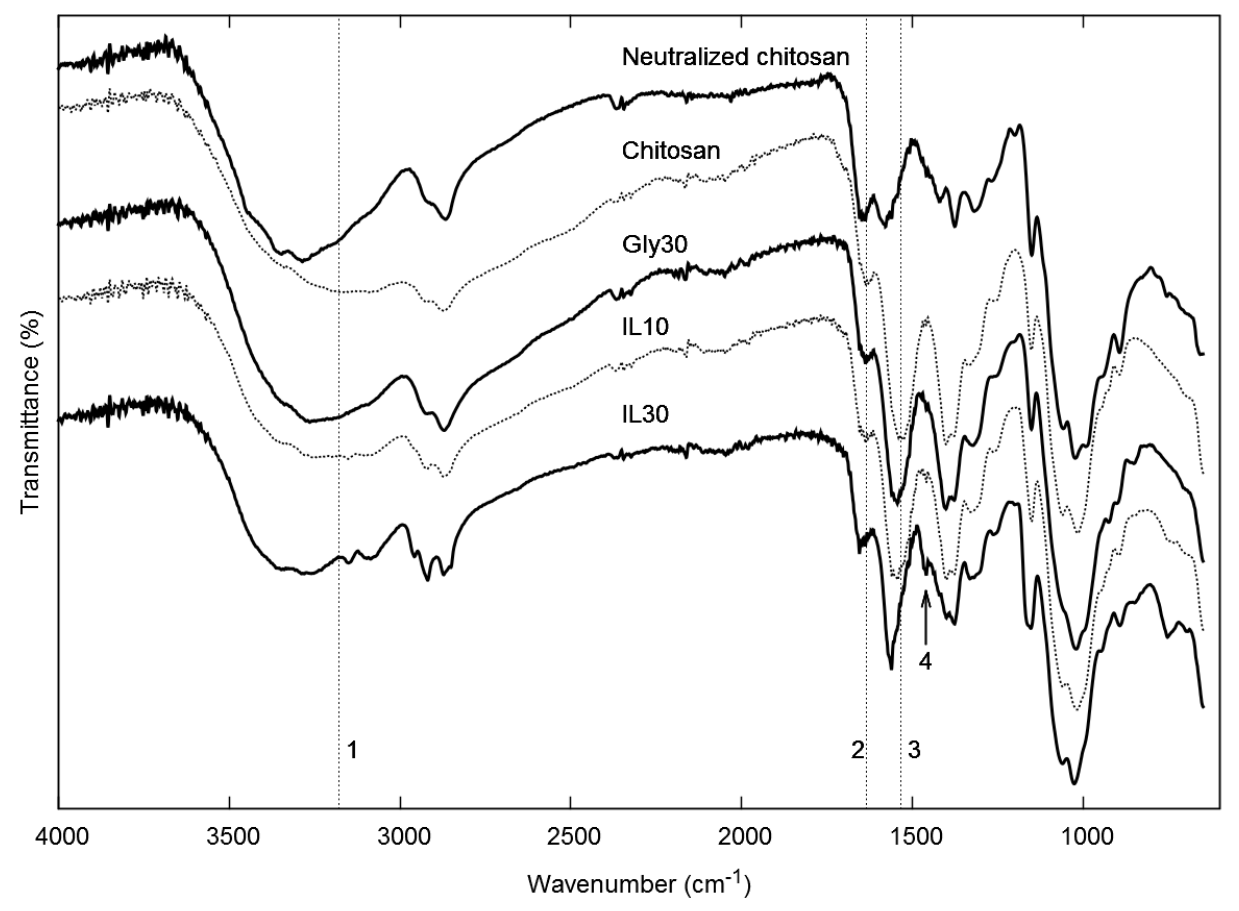

Figure 3: FTIR spectra of pure and plasticized chitosan. Neutralizedchitosan: this film was neutralized with $\mathrm{NaOH}$ and thoroughly washed before the measurement. The vertical lines help the reader identify the major vibrations of chitosan discussed in the text and their respective displacement with addition of plasticizer: 1 ) wide band at $\sim 3180 \mathrm{~cm}^{-1}, 2$ ) amide I band, and 3) amide II band. The arrow (4) identifies the band at $\sim 1460 \mathrm{~cm}^{-1}$ from the IL. 
to what was observed here for the amide II band of chitosan. Accordingly, Chen et al. (2011) described that both the hydroxyl oxygen and/or the amine nitrogen in chitosan interact with the protons in the $\mathrm{BMIM}^{+}$cation, disrupting the intra- and inter- molecular hydrogen bonding in chitosan. Based on these assumptions, the current results suggest that, due to the strong interaction chitosan/IL, a lower amount of $\mathrm{NH}_{2}$ groups would be available to interact with acetic acid, as seen for pure chitosan; therefore the amide II band would be less shifted than in pure chitosan, as seen in Fig. 3. This effect was not so evident in IL10 (due to the lower amount of plasticizer) or Gly30 (due to the non-ionic nature of glycerol), as seen by a smaller shift of the amide II band (see Table 2).

In summary, the better performance of IL as a plasticizer for chitosan is due to both a mechanism already seen for starch (that is, suppression of inter- and intra-molecular hydrogen bonds) (Sankri et al., 2010), as well as to the ionic interaction between both components. I thus proceeded to examine the effect of these plasticizers on biomimetic composites of chitosan and clay.

\subsection{Effect of plasticizers on biomimetic composites of chitosan}

As already mentioned, brittleness is one of the main problems of the biomimetic composites, which usually display very high mechanical strength and barrier properties. The objective of the second part of this study was, therefore, to examine the effect of glycerol and IL on the mechanical and barrier properties of chitosan-based biomimetic composites. The plasticized composites were also characterized by SEM, XRD, and TGA. Table 3 displays the real amount of clay in each composite, as determined by TGA.

Figure 4 shows SEM micrographs of cryogenically fractured cross-sections of the composites. All of them presented a very similar structure, which differed noticeably from the ones of the unfilled polymers: the composites present a layered structure caused by the highly oriented clay platelets aligned parallel to the film surface and by the alternating assembly of inorganic and organic layers (Walther et al., 2010; Yao et al., 2010). As already explained by those authors, this arrangement arises due to the intercalation of polymer molecules in the interlayer of clay platelets.(Walther et al., 2010; Yao et al., 2010) According to Darder et al. (2003), the intercalation of chitosan as a monolayer leads to an increase in the basal spacing of montmorillonite from $\sim 1,2 \mathrm{~nm}$ to $\sim 1,45 \mathrm{~nm}$, while a bilayer would show a basal spacing of $\sim 2,1 \mathrm{~nm}$. Figure 5 shows the XRD spectra of montmorillonite powder and films of the biomimetic composites (BNC). The basal spacings 
Table 3: Dimensions of the interlayer spacing before and after chitosan intercalation in the clay, and crystal size of chitosan.

\begin{tabular}{c|c|c|c|c}
\hline Material & $\begin{array}{c}\text { Clay } \\
\text { amount }(\%)\end{array}$ & \multicolumn{3}{|c}{$d_{h k l}(\mathrm{~nm}) /(\mathrm{hkl})$} \\
& Reflections from the clay & $(100)$ Reflection from chitosan \\
\hline Chitosan & 0 & - & - & 0,43 \\
Na-MMT* & 100 & $1,28 /(001)$ & $0,62 /(002)$ & - \\
Chi_ M $_{\text {Gly30_M }}$ & 59 & $2,44 /(001)_{t}$ & $1,46_{s h} /(001)_{m}$ & 0,51 \\
IL10_M & 72 & $2,40 /(001)_{t}$ & $1,42 /(001)_{m}$ & 0,53 \\
IL30_M & 66 & $2,38 /(001)_{t}$ & $1,44 /(001)_{m}$ & 0,47 \\
\hline
\end{tabular}

Subscripts "t" and "m" correspond to chitosan intercalated as a tri- or a monolayer, respectively; "sh" means it is a shoulder to the main peak.

* Pristine montmorillonite.

and other $d_{h k l}$ reflections are summarized in Table 3. For all BNC, chitosan is clearly present in a mixed situation: either as a monolayer or at a much higher basal spacing $(\sim 2,4 \mathrm{~nm})$, even higher than that caused by the intercalation as a bilayer. This value could either refer to the intercalation of chitosan as a trilayer, or in a more disorganized conformation. Considering, however, that the typical (100) reflection appears in all cases (at $2 \theta \sim 22^{\circ}$ )(Kittur et al., 2003; Matet et al., 2013), I assume that chitosan is present in a well ordered, crystalline phase between the clay platelets; therefore, the hypothesis of a trilayer seems more plausible.

Interestingly, the effect of the plasticizer follows the same trend as that already discussed in the previous section. For the unplasticized BNC, most of the chitosan is intercalated as a trilayer (the peak at $\sim 7,2^{\circ}$ is only a shoulder of the main peak) while for the plasticized ones the intensity of this reflection increases in the order Gly30<IL10<IL30. Clearly, the better the plasticizing effect is, the stronger the favouring of chitosan intercalation as a monolayer. Both glycerol (Chivrac et al., 2010; Tang et al., 2008) and imidazolium-based ionic liquids (Gilman et al., 2002; Kim et al., 2006) have been shown to intercalate easily into the clay layers, either through hydrogen bonding or ionic interactions with the clay. As they also interact with chitosan, they work as a kind of "promoter" for the ordered, monolayer intercalation of chitosan, by simultaneously breaking intermolecular bonds in the chitosan matrix and favouring electrostatic interactions with clay. Moreover, Lavorgna et al. (2010) have recently shown that conventional (that is, 


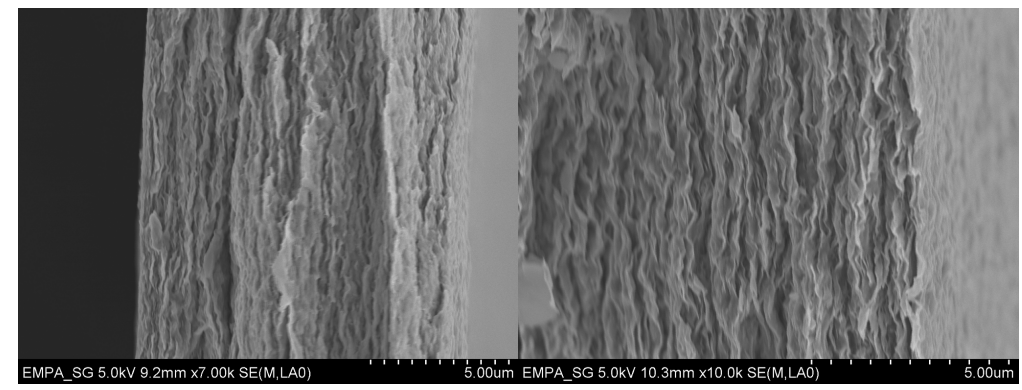

(a)

(b)

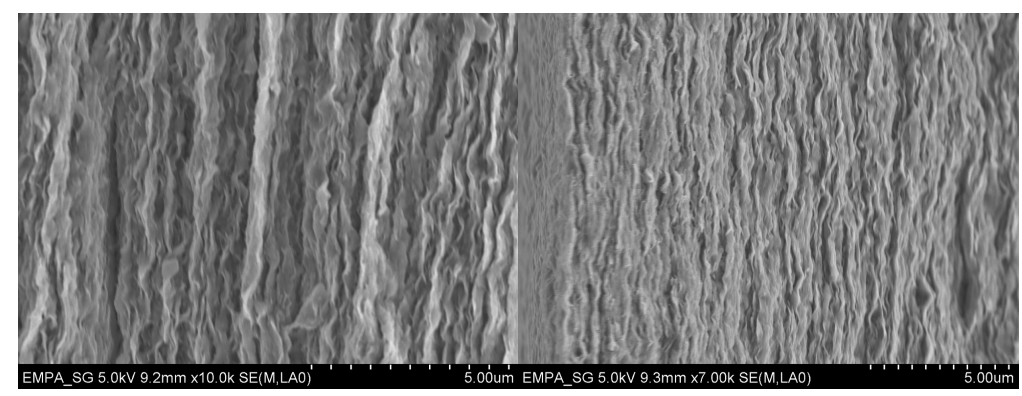

(c)

(d)

Figure 4: SEM images of the cross section of the base chitosan/clay composite (Chi_M) (a), Gly30_M (b), IL10_M (c), and IL30_M (d). 
with low clay loading) nanocomposites of chitosan and clay have a better intercalated structure when glycerol is added as a plasticizer. The reason is thought to be the decrease of hydrogen bonding interaction between chitosan and montmorillonite edges (which causes flocculation) due to the preferential interaction between glycerol and clay edges, what hindered flocculation and facilitated chitosan intercalation.(Lavorgna et al., 2010) Tang et al. (2008) also found out small amounts of glycerol promoted exfoliation of clay platelets in a starch matrix (in conventional nanocomposites). So far, no studies have been reported where ionic liquids are used as plasticizers but, given the good miscibility of IL with clays (Gilman et al., 2002; Kim et al., 2006), it may be assumed that IL would perform in a similar way as glycerol, or even better due to their ionic character. In fact, the latter seems to be the case in the present study: as seen in Figure 5, the ratio of monolayer to trilayer intercalation in IL30 is much higher than in Gly30, meaning that there was a better dispersion of clay in the former composite.

As already mentioned, the main reason for plasticizers incorporation was the improvement of ductility of such composites. Table 4 summarizes the mechanical properties of BNC. A similar trend was observed here as for the polymers (Table 1), that is: IL is more efficient in changing the mechanical properties than glycerol. The properties of the BNC with glycerol, in fact, are only marginally different from those without any plasticizer. Although the absolute value for the strain at break is always small, it is important to stress that the composite with IL is twice as ductile as the base composite. Moreover, as seen is Figure S2c, there is a clear change in mechanical behaviour between the base composite (Chi_M) and the one plasticized with ionic liquid (IL30_M): the latter shows a plastic deformation regime, while the former breaks in a purely elastic regime. The effect of plasticizer in such composites was in any case expected to be smaller than for the polymer, because the properties are controlled by the main component, the clay. The fact that such a small amount of ionic liquid (ca. $12 \%$ of the final composition) contributed to a doubling of the ductility and to a change from elastic to plastic regime is noteworthy. Furthermore, in a typical situation of such composites being used as barrier layers or barrier coatings for packaging applications, bending and/or tearing stresses will be more relevant than tensile stresses. Under such conditions, even smaller increases in the measured elongation at break under tensile load would translate into a clear improvement in performance. The Supporting Information Videos 4 and 5 show the behaviour of two films: Chi_M $(\epsilon \sim 1,5 \%)$ and IL30_M $(\epsilon \sim 5,0 \%$, extra plasticized by equilibrating the film at $50 \% \mathrm{RH})$. There is an obvious difference in the performance of these two extreme cases, where the extra 


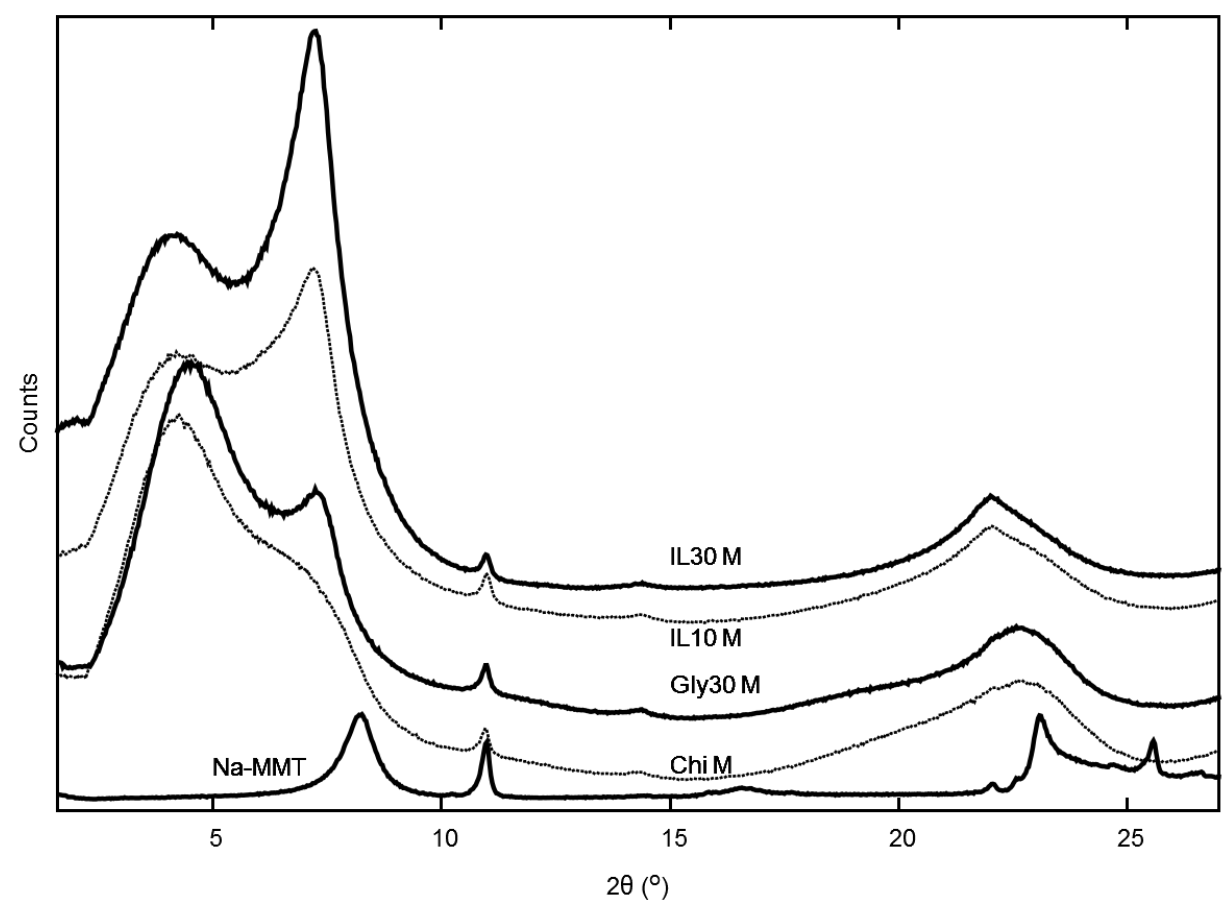

Figure 5: XRD spectra of pristine montmorillonite (Na-MMT) and biomimetic composites. The peak at $2 \theta=11^{\circ}$ is from talc, used as a reference. Other main peaks: chitosan intercalated as a trilayer $\left(4,2^{\circ}\right)$ or as a monolayer $\left(7,2^{\circ}\right)$, basal spacing of the pristine clay $\left(8,2^{\circ}\right)$, and $(100)$ reflection of chitosan $\left(22^{\circ}\right)$. Table 3 shows the corresponding $d_{h k l}$ for these reflections. 
Table 4: Mechanical and barrier properties of biomimetic nanocomposites.

\begin{tabular}{c|c|c|c|c|c}
\hline Sample & $E_{t}(\mathrm{MPa})$ & $\begin{array}{c}\sigma_{t}(\mathrm{MPa}) \\
(\text { Vacuum dried) }\end{array}$ & $\epsilon_{b}(\%)$ & $\begin{array}{c}\mathrm{P}_{W V} \\
*\end{array}$ & $\begin{array}{c}\mathrm{P}_{O_{2}} \text { at } 70 \% \mathrm{RH} \\
*\end{array}$ \\
\hline Chitosan & $3,6 \pm 0,7$ & $(1,2 \pm 0,2) \times 10^{2}$ & $18 \pm 8$ & $12 \pm 4$ & $21 \pm 0,1$ \\
Chi_M & $12 \pm 2^{a}$ & $(1,5 \pm 0,4) \times 10^{2, a}$ & $1,5 \pm 0,2^{a}$ & $2,9 \pm 1,0^{a}$ & $3,1 \pm 0,3^{a}$ \\
Gly30_M & $10 \pm 3^{a}$ & $(1,0 \pm 0,2) \times 10^{2, b}$ & $1,1 \pm 0,3^{a}$ & $4,2 \pm 0,2^{a b}$ & $8,4 \pm 3,3^{b}$ \\
IL10_M & - & - & - & $5,1 \pm 1,0^{b}$ & $5,5 \pm 1,0^{a b}$ \\
IL30_M & $5,2 \pm 1,1^{b}$ & $71 \pm 13^{b}$ & $3,1 \pm 1,2^{b}$ & $4,3 \pm 1,2^{b}$ & $3,1 \pm 1,0^{a}$ \\
\hline
\end{tabular}

* Units: $\mathrm{P}_{W V}$ in $\left[10^{-14} \mathrm{~kg} \cdot \mathrm{m} /\left(\mathrm{m}^{2} \cdot s \cdot \mathrm{Pa}\right)\right]$, and $\mathrm{P}_{\mathrm{O}_{2}}$ in $\left[10^{-20} m^{3} \cdot m /\left(m^{2} \cdot s \cdot P a\right)\right]$.

Superscripts "a, b" identify samples (in the same column) which do not show significant differences for the corresponding property at $p<0,05$.

The sample "Chitosan" was not included in this statistical analysis.

plasticized film afforded bending/tearing loads without integrity loss. It is then clear that only small increases in ductility are needed to transform the brittle biomimetic composites into manipulatable materials.

Last, Table 4 shows the barrier properties of the BNC in comparison with chitosan. As expected, the unplasticized composite displayed much better properties than chitosan $\left(\sim 80 \%\right.$ decrease in $P_{\mathrm{H}_{2} \mathrm{O}}$ and $\sim 85 \%$ decrease in $P_{\mathrm{O}_{2}}$ ). Both the absolute value of the permeability as well as the percent reduction are in accordance to those found by me and other authors for polymers such as PLA, nanofribillate cellulose and polyvinylalcohol. (Boesel et al., 2013; Liu et al., 2011; Walther et al., 2010) Regarding the plasticized composites, they showed in general higher permeability values than the base composite, although the pattern was different for the two permeants. The best performance was the one of the IL30_M composite: no difference in oxygen permeability was found and, although the water vapour permeability was significantly higher than that of Chi_M, it was still about three times better than that of pure chitosan. It is interesting to note that the barrier properties of the plasticized composites can be associated neither with the plasticization efficiency as seen for the polymer (cf. IL30_M versus Gly30_M), nor with the amount of plasticizer (cf. IL30_M versus IL10_M). The trend in permeability data of the plasticized composites agrees very well with the trend seen in the intercalation of chitosan into the clay layers, specially for the oxygen permeability (see Figure 5): IL30_M, in which the 
monolayer intercalation of chitosan was strongly favoured, displayed the best permeability data of the three plasticized composites, while Gly30_M (with a higher content of more disorganized or trilayer intercalation of chitosan) displayed the worst. It is clear that the clay plays the major role in determining the barrier properties of biomimetic composites; even the addition of plasticizers that strongly decrease the barrier performance of the pure polymer (cf. Table 2) does not hinder the performance of the composites. Moreover, the composite structure induced by the plasticizers seemed to play a major role, whereas the role of the type or amount of the plasticizer was minor. Presumably, by using other plasticizers that could promote an even higher monolayer intercalation of chitosan, the barrier properties of plasticized composites could match or even surpass that of the base one, where the amount of monolayer intercalation was lower.

\section{Conclusion}

In this article, an imidazolium-based ionic liquid was shown to be an effective plasticizer for chitosan, due to the ionic interactions with the amine groups of chitosan. This efficacy was demonstrated in terms of mechanical and barrier properties, as well as in the decrease of the $T_{g}$. In all cases, the ionic liquid performed much better than the typical plasticizer glycerol. This increased effectiveness was also seen for plasticized biomimetic nanocomposites of chitosan and clay. Analogous to the plasticized chitosan films, composites plasticized with ionic liquid displayed a higher elongation than the base one, even though the total amount of plasticizer in the formulation was small. This effect of ionic liquid on the mechanical properties was attributed to a different conformation of chitosan in the interlayer of the clay, namely, to a favouring of monolayer intercalation to the detriment of a more disorganized or trilayer intercalation. On the other hand, the plasticizers played a minor role in determining the barrier properties of the composites, which were mainly controlled by the clay. Therefore, the incorporation of ionic liquid is an effective method of increasing the ductility of biomimetic composites without sacrificing their excellent barrier properties. The absolute improvement in ductility achieved in this work represented a twofold increase of this property, although the absolute difference was a modest one. Given that, for a barrier coating or barrier layer, the ductility and flexibility are much more important properties than extremely high strength, the strategy used herein is a first step towards the improvement of the general properties of biomimetic composites. The method and composition may still be optimized (e. g., with other ionic liquids) to pave the 
way for achieving strong, ductile and low permeability biomimetic composites. Such composites may then be applied as barrier coatings to flexible substrates without the risk of cracking under small deformations.

\section{Materials and Methods}

\subsection{Materials}

The chitosan used in this work was acquired from Sigma-Aldrich. It has a viscosity of $400 \mathrm{mPa} \mathrm{s}$ (measured at $20{ }^{\circ} \mathrm{C}$ in $1 \%$ acetic acid) and a deacetylation degree of approximately $80 \%$. The sodium montmorillonite (Dellite LVF) was supplied by Laviosa Chimica Mineraria S.p.A., Italy. The ionic liquid (1-butyl-3-methyl-imidazolium chloride, hereafter referred to as BMIM-Cl or IL, see schema below) was acquired from Sigma-Aldrich. Other reagents were of reagent-grade and used as received.

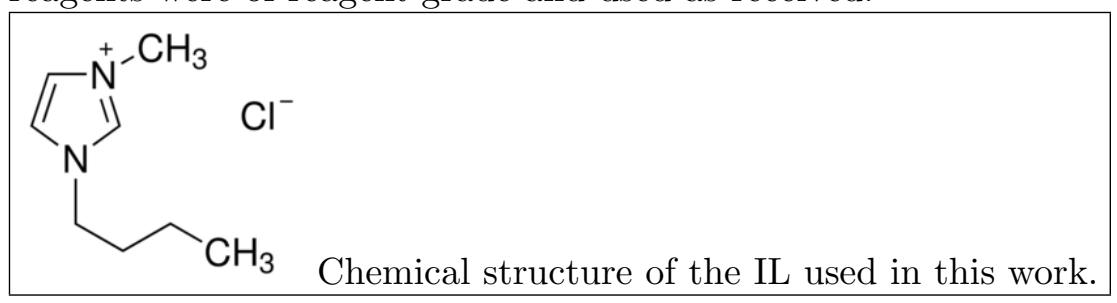

\subsection{Preparation and application of composites}

Chitosan was dissolved in acetic acid at a $1 \%$ concentration (mass/volume). When required, plasticizers (IL or glycerol) were added to this solution at $10 \%$ or $30 \%$ relative to the chitosan mass. Montmorillonite was dispersed in distilled water at a 0,5\% concentration (mass/volume). The chitosan or chitosan/plasticizer solution was then slowly added to the clay dispersion, and the mixture was stirred overnight and ultrasonicated to promote the intercalation of polymer and plasticizers into the clay layers. The mixture was then poured over petri-dishes to prepare films analogous to the ones reported by Yao et al. Yao et al. (2010) Pure and plasticized polymer films were also prepared by solvent-casting in a similar way, except that no clay was added to the solution. The excess solvent was initially dried at room temperature; residual solvent was then removed by vacuum-drying for $24 \mathrm{~h}$ at $60{ }^{\circ} \mathrm{C}$ temperature. The prepared films had thicknesses in the range of $40 \mu \mathrm{m}$ to $50 \mu \mathrm{m}$ (pure and plasticized polymer films), and of $15 \mu \mathrm{m}$ to $18 \mu \mathrm{m}$ (pure and plasticized composite films). The following polymer films were prepared: chitosan, chitosan containing $30 \%$ glycerol (Gly30), and chitosan containing $30 \%$ or $10 \%$ of IL (IL30 and IL10, respectively). The 
composites with approximately $60 \%$ of clay were named by adding the suffix "M" (from montmorillonite) to the name of the polymer, e. g., "Chi_M" was the composite of chitosan and clay, "IL30_M" was the composite of IL30 and clay, and so on.

\subsection{Characterization}

Fourier-transformed infra-red spectroscopy (FTIR) was performed with the Attenuated Total Reflectance (ATR) module. Both specimens and background spectra were collected at a $2 \mathrm{~cm}^{-1}$ resolution by adding 64 spectra.

Scanning electron microscopy (SEM) was performed in a Hitachi S-4800 instrument, using an acceleration voltage of between $2 \mathrm{kV}$ and $10 \mathrm{kV}$. Before observation, specimens were coated with a thin $(\sim 5 \mathrm{~nm})$ gold/palladium layer in a Leica ACE600 sputtering machine.

Water vapour permeability $\left(\mathrm{P}_{\mathrm{H}_{2} \mathrm{O}}\right)$ was measured according to ASTM Standard E-96 using the "water method". The area of the films (at least three per sample) was adjusted by using aluminium masks with the desired area. Tests were carried out at an external relative humidity of $50 \%$ and temperature of $23^{\circ} \mathrm{C}$.

Oxygen permeability $\left(\mathrm{P}_{\mathrm{O}_{2}}\right)$ was measured with a Mocon OxTran 1/50 instrument, at $23{ }^{\circ} \mathrm{C}$ and three different relative humidities $(0,50 \%$, and $70 \%$ ). The area of the films (at least three per sample) was adjusted by using aluminium masks with the desired area.

X-ray diffraction (XRD) was performed in a Bruker AXS D8 Advance apparatus, using Co $\mathrm{K} \alpha$ radiation $(1,79 \mathrm{~nm})$. The conditions used for all specimens were: scanning between $1,9^{\circ}$ and $60^{\circ}(2 \theta)$, with a step size of $0,03^{\circ}(2 \theta)$ and $10 \mathrm{~s} / \mathrm{step}$. Powder samples were analysed with the texture technique in order to improve the intensity of $00 l$ reflections of clay layers. Films were directly glued on the substrate with vaseline. Talc (with three main reflections at $11,0^{\circ}, 21,9^{\circ}$, and $33,4^{\circ}$ ) was used as an internal standard to adjust the position of the reflections of all specimens.

Thermogravimetric analysis (TGA) was performed in a Netzsch TG209F1 apparatus to determine the real amount of clay in each composite. Isothermal experiments were conducted as follows: $20 \mathrm{~min}$ at $110^{\circ} \mathrm{C}$ (to remove any residual water), and $60 \mathrm{~min}$ at $800^{\circ} \mathrm{C}$ (to remove polymer and plasticizers). The experiments were performed in triplicate.

Mechanical tests were performed in tensile mode with dog-bone specimens (4 mm width and $25 \mathrm{~mm}$ parallel length) in a Zwick Z100 equipped with a $100 \mathrm{~N}$ load cell. Due to the brittleness of most specimens, only one loading speed was used $\left(8,33 \times 10^{-5} \mathrm{~m} / \mathrm{s}\right.$, corresponding to $\left.5 \mathrm{~mm} / \mathrm{min}\right)$. Specimens (a minimum of 7 for each sample) were tested either immediately 
after vacuum-drying at $60{ }^{\circ} \mathrm{C}$ for $24 \mathrm{~h}$, or after equilibration at $50 \% \mathrm{RH}$ for one week. The following mechanical properties were determined: tensile strength $\left(\sigma_{t}\right)$, yield stress $\left(\sigma_{y}\right)$, tensile modulus $\left(E_{t}\right)$, and elongation at break $\left(\epsilon_{b}\right)$.

Dynamic mechanical thermal analysis (DMTA) was performed in a RS III Rheometrics System Analyzer from TA Instruments-Waters, LLC, USA. Tests were performed in tensile mode with a fixed frequency of $1 \mathrm{~Hz}$. In order to remove any residual water from the specimens, they were conditioned at $110{ }^{\circ} \mathrm{C}$ for $10 \mathrm{~min}$ before performing the experiments. Two types of test modes were used: strain sweep (from $0,001 \%$ to $1 \%$ ), in order to define the region of linear viscoelasticity, and temperature sweeps (from $0-250{ }^{\circ} \mathrm{C}$ ), in order to determine the glass transition temperature $\left(T_{g}\right)$.

\subsection{Statistics}

Statistical data analysis was performed with the "R" program and the "R-commander" package. (Fox, 2005; R Core Team, 2012) Shapiro-Wilk tests were used to test for the normality of the samples; afterwards differences in the central values of two samples were tested by using the Student $t$ test (for samples following the normal distribution) or the Mann-Whitney $U$ test (otherwise). One-way analysis of variance (ANOVA) was used to test for differences in means of groups of samples following the normal distribution, with Tukey Contrasts being subsequently used for the multiple comparisons of means. In case of non-normal samples, I applied the Kruskal-Wallis Rank Sum tests and Pairwise Wilcoxon Rank Sum tests, respectively.

\section{Acknowledgments}

The author thanks Dr. Michael Plötze (ETHZ, Switzerland) for allowing the use of the XRD apparatus, E. Falk for help with the preparation of films and with the WVTR measurements. Prof. L. Thöny-Meyer and Dr. H. S. Azevedo (QMUL, UK) are also acknowleged for a critical review of the manuscript.

\section{References}

Boesel, L. F., de Geus, M., Thöny-Meyer, L., 2013. Effect of PLA crystallization on the structure of biomimetic composites of PLA and clay. Journal of Applied Polymer Science 129, 1109-1116.

Bonderer, L. J., Studart, A. R., Gauckler, L. J., 2008. Bioinspired design and assembly of platelet reinforced polymer films. Science 319, 1069-1073. 
Chen, Q., Xu, A., Li, Z., Wang, J., Zhang, S., 2011. Influence of anionic structure on the dissolution of chitosan in 1-butyl-3-methylimidazoliumbased ionic liquids. Green Chemistry 13, 3446-3452.

Chivrac, F., Pollet, E., Dole, P., Avérous, L., 2010. Starch-based nanobiocomposites: Plasticizer impact on the montmorillonite exfoliation process. Carbohydrate Polymers 79, 941-947.

Darder, M., Colilla, M., Ruiz-Hitzky, E., 2003. Biopolymer-clay nanocomposites based on chitosan intercalated in montmorillonite. Chemistry of Materials 15, 3774-3780.

Epure, V., Griffon, M., Pollet, E., Avérous, L., 2011. Structure and properties of glycerol-plasticized chitosan obtained by mechanical kneading. Carbohydrate Polymers 83 (2), 947-952.

Espinosa, H. D., Rim, J. E., Barthelat, F., Buehler, M. J., 2009. Merger of structure and material in nacre and bone - Perspectives on de novo biomimetic materials. Progress in Materials Science 54 (8), 1059-1100.

Fox, J., 2005. The R Commander: A basic statistics graphical user interface to R. Journal of Statistical Software 14, 1-42.

Gilman, J. W., Awad, W. H., Davis, R. D., Shields, J., Harris Jr, R. H., Davis, C., Morgan, A. B., Sutto, T. E., Callahan, J., Trulove, P. C., DeLong, H. C., 2002. Polymer/layered silicate nanocomposites from thermally stable trialkylimidazolium-treated montmorillonite. Chemistry of Materials 14, 3776-3785.

Jeon, Y., Sung, J., Seo, C., Lim, H., Cheong, H., Kang, M., Moon, B., Ouchi, Y., Kim, D., 2008. Structures of ionic liquids with different anions studied by infrared vibration spectroscopy. Journal of Physical Chemistry B 112, 4735-4740.

Kim, N. H., Malhotra, S. V., Xanthos, M., 2006. Modification of cationic nanoclays with ionic liquids. Microporous and Mesoporous Materials 96, $29-35$.

Kittur, F. S., Kumar, A. B. V., Tharanathan, R. N., 2003. Low molecular weight chitosans - preparation by depolymerization with Aspergillus niger pectinase, and characterization. Carbohydrate Research 338, 1283-1290. 
Lavorgna, M., Piscitelli, F., Mangiacapra, P., Buonocore, G. G., 2010. Study of the combined effect of both clay and glycerol plasticizer on the properties of chitosan films. Carbohydrate Polymers 82 (2), 291-298.

Liu, A., Walther, A., Ikkala, O., Belova, L., Berglund, L. A., 2011. Clay nanopaper with tough cellulose nanofiber matrix for fire retardancy and gas barrier functions. Biomacromolecules 12, 633-641.

Matet, M., Heuzey, M.-C., Pollet, E., Ajji, A., Avérous, L., 2013. Innovative thermoplastic chitosan obtained by thermo-mechanical mixing with polyol plasticizers. Carbohydrate Polymers 95 (1), 241-251.

Ning, W., Xingxiang, Z., Haihui, L., Na, H., 2009. Ionically conducting polymers based on ionic liquid-plasticized starch containing lithium chloride. Polymers \& Polymer Composites 18 (1), 53-58.

Osman, Z., Arof, A. K., 2003. FTIR studies of chitosan acetate based polymer electrolytes. Electrochimica Acta 48, 993-999.

Priolo, M., Gamboa, D., Grunlan, J., 2010a. Transparent clay-polymer nano brick wall assemblies with tailorable oxygen barrier. ACS Applied Materials and Interfaces 2 (1), 312-320.

Priolo, M. A., Gamboa, D., Holder, K. M., Grunlan, J. C., 2010b. Super gas barrier of transparent polymer-clay multilayer ultrathin films. Nano Letters 10 (12), 4970-4974.

Quijada-Garrido, I., Iglesias-González, V., Mazón-Arechederra, J. M., Barrales-Rienda, J. M., 2007. The role played by the interactions of small molecules with chitosan and their transition temperatures. glass-forming liquids: 1,2,3-propantriol (glycerol). Carbohydrate Polymers 68, 173-186.

R Core Team, 2012. R: A Language and Environment for Statistical Computing. R Foundation for Statistical Computing, Vienna, Austria, ISBN 3-900051-07-0.

URL http://www.R-project.org/

Sankri, A., Arhaliass, A., Dez, I., Gaumont, A. C., Grohens, Y., Lourdin, D., Pillin, I., Rolland-Sabaté, A., Leroy, E., 2010. Thermoplastic starch plasticized by an ionic liquid. Carbohydrate Polymers 82, 256-263.

Tang, X., Alavi, S., Herald, T. J., 2008. Effects of plasticizers on the structure and properties of starch-clay nanocomposite films. Carbohydrate Polymers 74, 552-558. 
Walther, A., Bjurhager, I., Malho, J.-M., Pere, J., Ruokolainen, J., Berglund, L. A., Ikkala, O., 2010. Large-area, lightweight and thick biomimetic composites with superior material properties via fast, economic, and green pathways. Nano Letters 10 (8), 2742-2748.

Yao, H.-B., Tan, Z.-H., Fang, H.-Y., Yu, S.-H., 2010. Artificial nacre-like bionanocomposite films from the self-assembly of chitosan-montmorillonite hybrid building blocks. Angewandte Chemie, International Edition 49 (52), 10127-10131.

Zollfrank, C., Weinkamer, R., Studart, A., 2012. Bioinspired materials: An emerging field of multidisciplinary research. Bioinspired, Biomimetic and Nanobiomaterials 1, 63-66. 